\title{
Neural Basis of Semantic and Syntactic Interference in Sentence Comprehension
}

\author{
Yi G. Glaser ${ }^{1}$, Randi C. Martin 1 , Julie A. Van Dyke ${ }^{2}$, A. Cris Hamilton ${ }^{1}$, and Yingying Tan ${ }^{1}$ \\ ${ }^{1}$ Rice University, Department of Psychology, 6100 Main Street, Houston, TX 77005 \\ ${ }^{2}$ Haskins Laboratories, 300 George Street, New Haven, CT 06511-6695
}

\section{Abstract}

According to the cue-based parsing approach (Lewis, Vasishth, \& Van Dyke, 2006), sentence comprehension difficulty derives from interference from material that partially matches syntactic and semantic retrieval cues. In a 2 (low vs. high semantic interference) $\times 2$ (low vs. high syntactic interference) fMRI study, greater activation was observed in left BA 44/45 for high versus low syntactic interference conditions following sentences and in BA 45/47 for high versus low semantic interference following comprehension questions. A conjunction analysis showed BA45 associated with both types of interference, while BA47 was associated with only semantic interference. Greater activation was also observed in the left STG in the high interference conditions. Importantly, the results for the LIFG could not be attributed to greater working memory capacity demands for high interference conditions. The results favor a fractionation of LIFG wherein BA45 is associated with post-retrieval selection and BA47 with controlled retrieval of semantic information.

\section{Keywords}

fMRI; Left Inferior Frontal Gyrus; Sentence Comprehension; Interference; Working Memory

\section{Introduction}

During sentence comprehension, earlier information must often be linked with later information across a potentially unbounded amount of intervening material. Take as an example the sentence, in (1).

1. The manager who knows that the owner has too much work during the month of December hires a new assistant every year.

In this sentence, "manager" has to be linked as the subject of the verb "hires" across the intervening information in the relative clause. Theories of sentence parsing have assumed different memory mechanisms that support this linkage (also termed integration). Those that emphasize capacity (e.g., Just \& Carpenter, 1992; Gibson, 2000) postulate that earlier information needs to be retained in an activated state until it is successfully integrated with the rest of the sentence. According to this approach, the capacity for sentence

(C) 2013 Elsevier Inc. All rights reserved.

Corresponding author: Randi C. Martin, rmartin@ rice.edu, phone: 713-348-3417, fax: 713-348-5221.

Publisher's Disclaimer: This is a PDF file of an unedited manuscript that has been accepted for publication. As a service to our customers we are providing this early version of the manuscript. The manuscript will undergo copyediting, typesetting, and review of the resulting proof before it is published in its final citable form. Please note that during the production process errors may be discovered which could affect the content, and all legal disclaimers that apply to the journal pertain. 
comprehension is determined by the total amount of information that can be stored and processed simultaneously in working memory. However, several lines of evidence have recently challenged this approach, suggesting that the capacity for maintaining information is extremely limited (Cowan, 2001; McElree, 2006; Oberauer, 2002; see Van Dyke \& Johns, 2012 for a review). This calls into question the notion that an entire sentence could be maintained in active memory until all linguistically dependent constituents are integrated. An alternative approach de-emphasizes capacity in favor of a cue-driven associative retrieval mechanism, which compensates for a limited memory capacity by quickly restoring information into the focus of attention as it is needed (Lewis, Vasishth, \& Van Dyke, 2006; McElree, Foraker, \& Dyer, 2003; Van Dyke, 2007; Van Dyke \& McElree, 2011). A computational implementation (Lewis \& Vasishth, 2005) has demonstrated the feasibility of this approach, relying on an active memory containing only two chunks of information (i.e., the item currently needing to be integrated, and the current state of the parse.) In this parser, when a word is brought into the focus of attention, stored lexical knowledge about the word is retrieved, in the form of lexical frames that specify basic syntactic argument structure (cf. Lewis \& Vasishth, 2005). These frames are similar to the lexical frames in the unification models of Tabor and Hutchens (2004) and Vosse and Kempen (2000), however they are restricted to information about core arguments only (e.g., subject, direct object) and incorporate lexical-semantic restrictions on the types of nouns that can fill those argument slots. This information becomes the cues that will enable a word to be integrated with the previously processed lexical context. For example, in sentence (1), "manager" will be encoded as a bundle of syntactic and semantic features, such as singular noun, subject, and human, along with a prediction for an upcoming verb (See Lewis et al., 2006, for further details.) A similar set of features would be encoded for "owner" though the predicted slot for the verb would be filled once "has" was processed. When the focus of attention shifts to the verb "hires", it is identified as a singular tensed verb, which requires an animate subject of the type that can "hire" someone. These syntactic and semantic cues are combined to create a retrieval probe that will directly access all previously encoded information via a global matching associative mechanism (e.g., Clark \& Gronlund, 1996). ${ }^{1}$ Because the encoded properties of "manager" match the retrieval cues (i.e., it is a singular animate noun encoded as a subject, which could plausibly hire someone), it will be retrieved as a likely subject for "hires." However, "owner" also matches the retrieval probe because it is also the subject of a sentence and refers to a human hire-er. Even though "manager" provides a better match because of the open slot for the verb, "owner" will be retrieved in error on a high proportion of trials because of the high degree of partial match. This incorrect match creates interference for building the correct association between "manager" and "hires" and provides an alternative, non-capacity-based, explanation for why this sentence seems difficult to understand.

A recent study of Van Dyke (2007, see also Van Dyke \& McElree, 2011) provides empirical support for the presence of syntactic and semantic interference in sentence processing. Van Dyke manipulated the semantic and syntactic properties of the intervening noun phrases to create high and low interference conditions (i.e., high vs. low semantic interference $\times$ high vs. low syntactic interference). A set of examples is as follows:

\footnotetext{
${ }^{1}$ Global matching is a common feature of many models of recognition memory (e.g., Murdock, 1982; Gillund \& Shiffrin, 1984; Hintzman, 1988; Humphreys, Bain, \& Pike, 1989; Shiffrin \& Steyvers, 1997; Norman \& O'Reilly, 2003), and refers to the signaldetection process through which retrieval cues are compared with the contents of memory. They are referred to as "global" because recognition of a given item is determined both by the similarity of the retrieval probe to the target memorandum and also the similarity of the retrieval probe to all other items in memory. The comparison process occurs via direct, content-based matches to all items in memory, and is therefore speed invariant with respect to factors such as number of items in memory or hierarchical positioning among items. These factors will affect the accuracy of the match, however, and it is exactly this which gives rise to interference effects. Clark and Gronlund (1996) provide an accessible tutorial for the mathematics underlying this process. A more technical review is provided in Humphreys, Bain, \& Pike (1989).
} 
1. Low semantic-Low syntactic. The worker was surprised that the resident who was living near the dangerous warehouse was complaining about the investigation.

2. High semantic-Low syntactic. The worker was surprised that the resident who was living near the dangerous neighbor was complaining about the investigation.

3. Low semantic - High syntactic. The worker was surprised that the resident who said the warehouse was dangerous was complaining about the investigation.

4. High semantic - High syntactic. The worker was surprised that the resident who said the neighbor was dangerous was complaining about the investigation.

In all of these example sentences, the subject of the main clause "resident" needs to be integrated with the verb "complain" across the intervening relative clause. In the high syntactic interference conditions, the intervening noun matched the syntactic encoding of the target noun (they are both subjects), whereas in the low syntactic interference conditions, the noun was a prepositional object. In the high semantic interference conditions, the intervening noun was semantically plausible as the subject of the main verb whereas in the low semantic condition, it was not. Van Dyke (2007) reported longer reading times on the main clause verb in the high syntactic interference condition, replicating an earlier study (Van Dyke \& Lewis, 2003). She also reported longer reading times in the high as compared to the low semantic interference condition, though these appeared downstream from the critical verb.

Once interference is detected, some researchers assume that a control process is initiated that allows for selection among the competing alternatives (e.g., Badre \& Wagner, 2007; Thompson-Schill, D'Esposito, Aguirre, \& Farah, 1997). From a computational viewpoint, different mechanisms have been proposed for this control process, such as lateral inhibition between alternative choices (Hagoort, 2005; Howard, Nickels, Coltheat, \& Cole-Virtue, 2006) or the involvement of a "booster" mechanism which serves to amplify differences in the activation of alternative choices until a difference threshold is reached (Oppenheim, Dell, \& Schwartz, 2009). Regardless of the specific mechanism, brain regions that are involved in selecting representations should be more active for high versus low interference conditions. With respect to cognitive control, regions in the left inferior frontal gyrus (LIFG) appear to play a critical role. Findings from neuroimaging studies have shown that this region is more highly activated for high as compared to low control conditions in various tasks (Badre, Poldrack, Paré-Blagoev, Insler, \& Wagner, 2005; Kan \& Thompson-Schill, 2004a, 2004b; Schnur, Schwartz, Kimberg, Hirshorn, Coslett, \& Thompson-Schill, 2009; Thompson-Schill, D'Esposito, Aguirre, \& Farah, 1997; Ye \& Zhou, 2009a). For instance, Kan and Thompson-Schill (2004a) found that the LIFG (BA 44 \& BA 45) was more engaged when a naming task required selection from multiple competing names (e.g., a picture of a stove evokes many names, such as "stove", "oven", and "range") than when there was less competition due to high name agreement (e.g., a picture of a book evokes a single reliable response "book"). Similarly, Badre et al., (2005) found that a single region centered at BA $45(-54,21,12)$ was associated with selection demands in four different tasks, each of which required selection among alternatives activated via an automatic associative cue-based retrieval mechanism. They argued that a post-retrieval selection process was a necessary complement to automatic retrieval, since it rarely occurs that the right conjunction of cues is present to guarantee retrieval of only the goal relevant information (Badre \& Wagner, 2002; Fletcher, Shallice, \& Dolan, 2000; Moss et al., 2005; Zhang, Feng, Fox, Gao, \& Tan, 2004). This process is in contrast to a controlled retrieval process, which becomes necessary when desired semantic information is not automatically retrieved (Tomita, Ohbayashi, Nakahara, Hasegawa, \& Miyashita, 1999). Using a factor analysis method, Badre et al. (2005; Badre \& Wagner, 2007) identified a region in LIFG centered at BA $47(-45,27,-15)$, which was associated with top-down use of cues to bias 
the activation of necessary knowledge. Notably, this region dissociated from that centered in BA 45 in that it was not activated by the selection component derived from any of the four tasks in their study.

While Badre and colleagues (2005) argued that at least the post-retrieval selection process is domain general (see also Kan \& Thompson-Schill, 2004b, Snijders et al., 2008), the extent to which the LIFG is involved in cognitive control for all types of representations is under debate. For example, Hamilton and Martin (2005) reported a patient with LIFG damage who showed deficits in resolving interference of verbal information but not of spatial information, suggesting that this region is not involved in cognitive control of spatial information. Its role in processing competing syntactic representations in garden-path and other types of ambiguous sentences is well attested, however (Fiebach, Vos, \& Friederici, 2004; January, Trueswell, \& Thompson-Schill, 2009; Mason, Just, Keller, \& Carpenter, 2003; Rodd, Longe, Randall, \& Tyler, 2010). Also, studies of individuals with damage to the LIFG have revealed deficits in their ability to resolve lexical and syntactic ambiguities (Novick, Kan, Trueswell, \& Thompson-Schill, 2009; Vuong \& Martin, 2011). In all these studies, the LIFG is assumed to be involved in abandoning a preferred parse in favor of selecting a dispreferred parse (Novick, Trueswell, \& Thompson-Schill, 2005; Vuong \& Martin, 2011). These processes may involve controlled retrieval of alternative syntactic frames, and possibly the reconstruction of entire semantic interpretations, so the contribution of syntactic vs. semantic processing is unclear.

The current study contrasts with this work in examining unambiguous sentences, where the need for cognitive control relates to resolving competition from sentence elements that partially match syntactic and semantic retrieval cues, in a design that does not confound the two types of conflict. In addition, unlike much work with unambiguous sentences (Friederici, Ruschmeyer, Hahne, \& Fiebach, 2003; Ni et al., 2000; Ye \& Zhou, 2009a), only plausible sentences were used, which will avoid the possibility that neural activation reflects checking processes that are involved in detecting anomalies (cf., Brennan et al., 2012). These methods will allow a more direct assessment of whether particular regions in LIFG are specialized for processing particular types of linguistic information, or are sensitive to specific types of interference, during normal sentence processing. Moreover, the focus on interference resolution differentiates the present study from many others that have examined the relation of LIFG activation to working memory demands. In line with the evidence for involvement of the LIFG in standard verbal working memory paradigms (e.g., Braver et al., 1997), many have suggested that activation of the LIFG during sentence processing results from capacity demands (e.g., Just et al., 1996). In the current study, the sentences in the high and low semantic interference conditions (like those in sentences 1 vs. 2 and 3 vs. 4 above) were matched in terms of syntactic structure and in terms of the number of intervening words and unintegrated elements between the subject and main verb. Thus, to the extent that LIFG activation is greater in the high than low semantic interference conditions, the effect would be more readily attributed to interference resolution rather than capacity demands.

\section{Method}

\subsection{Subjects}

Twenty-four undergraduate students at Rice University were recruited as subjects through a web-based experiment scheduling system for a pilot behavioral study that was used to determine presentation times for the experimental sentences. Subjects received $1 \mathrm{credit}$ toward the fulfillment of classroom experiment participation requirements for one-hour of participation. 
Fourteen different subjects were recruited for the fMRI experiment through email announcements to undergraduate and graduate students at Rice University. Subjects were screened using a detailed questionnaire to ensure that they had no history of neurological or psychiatric problems. Subjects were compensated with $\$ 30$ for a 1.5 hour-participation. Data from two subjects were excluded due to uncorrectable head movement. Half of the subjects whose data were included in the analyses were female.

All subjects in the two experiments were native English speakers. In addition, all subjects were right-handed and had normal or corrected-to-normal vision. Informed consent was obtained from each subject in accordance with the guidelines and approval of the Rice University Institutional Review Board.

\subsection{Materials}

The stimuli included 480 critical sentences (120 sets of 4 conditions) and 120 filler sentences. For each sentence, a comprehension question was also constructed.

The critical sentences were simplified versions of those used by Van Dyke (2007). Each sentence contained a main clause and a relative clause that separated the subject and verb of the main clause. The four conditions represented a 2 (semantic interference: low vs. high) $\times$ 2 (syntactic interference: low vs. high) design (see Table 1 for examples). As in the Van Dyke (2007) materials, in the low semantic interference conditions, the intervening noun was implausible as the agent of the main verb whereas in the high semantic interference conditions it was plausible. In the low syntactic interference conditions, the intervening noun was a prepositional object whereas in the high syntactic interference conditions, it was a subject. These four sentences that have the same main clause but belong to different conditions, depending on the properties of the intervening nouns, are referred to as a set. These 480 items were divided into four lists according to a Latin Square design, so that each list only included 30 sentences for each condition (i.e., 120 critical sentences in each list), and no two sentences in a set occurred in the same list. Thus each subject only read one list of critical sentences and all of the filler items ( 240 sentences in total; target-filler ratio is 1:1). The comprehension questions for the four conditions of each set were identical, and always asked about the subject of the verb in the main clause (see Table 1 for examples). In this way we could examine whether participants made the correct subject-verb association or were distracted by the interfering information.

Filler sentences prevented subjects from intentionally ignoring the intervening relative clause and avoiding the interference. These sentences were constructed with various syntactic structures, including the same structures as the experimental sentences, object or subject extracted relative clause sentences, simple one-clause sentences, and conjoined sentences (see Appendix A for examples). More importantly, the comprehension questions for fillers asked about information from different regions of the sentences, so that if subjects adopted a strategy of avoiding the interference by only attending to the subject of the main clause, they would have trouble answering those questions.

All sentences were presented visually phrase by phrase (five-six phrases for each sentence) at the center of a computer screen (see Appendix A for all target sentences). Table 1 provides a breakdown of the phrasal units for the target sentences, with separate presentation units for the head noun (first phrase) and main clause verb (phrase 5). The embedded relative clauses were presented in three phrases including one with the relative pronoun and embedded verb (phrase 2), either a prepositional phrase (in the two low syntactic conditions) or embedded sentence (in the two high syntactic conditions) (phrase 3), and an adverbial phrase (phrase 4). Following the main clause verb (phrase 5), there was a spillover region that was either an adverbial phrase or the patient of the main clause verb (phrase 6). The 
same target and filler sentences were used in the pilot experiment and the neuroimaging experiment except that all target sentences consisted of six phrases in the fMRI experiment while some target sentences did not include the sixth phrase in the pilot study (i.e., when the main clause verb was an intransitive verb). Filler sentences that were structurally similar to the experimental sentences were divided into the same phrasal units as the latter. A similar scheme was used to delimit phrases for the other types of filler sentences, with phrases for head nouns, main verbs, adverbial and prepositional phrases, and embedded noun and verb phrases. All questions were presented visually as a single unit. Presentations and responsetime measurements were controlled by the PsyScope software package (Cohen, MacWhinney, Flatt, \& Provost, 1993).

\subsection{Procedure}

In the pilot behavioral experiment, subjects completed practice trials with sentences that would not appear in the actual task but had similar syntactic structures as the experimental and filler sentences. Each trial started with the first phrase of the sentence. Subjects were asked to push the spacebar to move to the next phrase once they finished reading. Each new phrase was presented immediately after the button push. The same procedure repeated until each sentence was over. Then a comprehension question appeared and subjects were instructed to verbally respond to the question as quickly and as accurately as possible. Following the verbal response, the question disappeared and a fixation cross (+) was presented on the screen until subjects pressed the spacebar again to start the next trial. The reading times for all phrases were recorded by the PsyScope software package. The verbal response times for the comprehension questions were also recorded by PsyScope with an embedded microphone. Additionally, the accuracy of the verbal responses was recorded by the experimenter who sat in during the pilot sessions.

For the fMRI experiment, subjects were told that they would see sentences presented phraseby-phrase in the center of the screen and that they should comprehend these sentences, as they would be asked questions about their meaning following the sentence. They then completed 12 practice trials outside the scanner. The presentation format was similar to the pilot behavioral experiment except that each phrase was presented for a fixed duration. These presentation durations were determined by the phrase reading times collected in the pilot experiment, and will be listed in the results section. The total presentation duration for each sentence was 7.1s. Following the last phrase, a fixation cross (+) was presented for $400 \mathrm{~ms}$. Then, a comprehension question was presented for $4 \mathrm{~s}$. In the scanner, the experiment employed a rapid-presentation event-related design. The intervals between trials and the intervals between each sentence and its question were both jittered (average rate of $2.5 \mathrm{~s}$ ), so that the hemodynamic signal changes driven by the sentences and the comprehension questions could be analyzed separately. A fixation cross remained on the screen during the jittered intervals. Subjects were instructed to comprehend the phrases while they were presented and asked to verbally respond to the questions as quickly and as accurately as possible. The verbal responses in the scanner were recorded by a MRI compatible microphone (Or-Yehuda, Israel; http://www.optoacoustics.com/) and exported to the Audacity audio recording software. Accuracy was coded offline. The response times were calculated relative to the onsets of comprehension questions. The timings were determined by examining the digitally recorded waveforms in Audacity. The entire fMRI scan consisted of 10 runs, which were each comprised of 3 target sentences from each condition and 12 filler sentences. As such, there were 24 trials in each run, and each run lasted approximately 7 minutes. 


\subsection{Image Acquisition and Analysis}

The scanning was conducted at University of Texas Medical School at Houston on a Philips 3T scanner using an eight-channel head gradient coil. At the beginning of each run, there was a 10s fixation to allow for stability in magnetization. At the end of each run, there was a $14 \mathrm{~s}$ fixation to compensate for the delay of the hemodynamic response. Anatomical images were acquired first, using a sagittal MP-RAGE T1-weighted sequence with a voxel size of . $9375 \times .9375 \times 1 \mathrm{~mm}\left(\mathrm{TR}=8.44 \mathrm{~ms} ; \mathrm{TE}=3.90 \mathrm{~ms}\right.$; flip angle $\left.=8^{\circ}\right)$. Functional images were acquired using an echo-planar sequence $\left(\mathrm{TR}=2500 \mathrm{~ms}\right.$; $\mathrm{TE}=30 \mathrm{~ms}$; flip angle $=90^{\circ}$; voxel size $=2.75 \times 2.75$ in-plane resolution). During each functional run, 176 sets of 40 contiguous 3-mm thick axial images were acquired. Visual stimuli were projected onto a screen using an LCD projector and viewed through a mirror attached to the head coil.

Imaging data were analyzed using the AFNI analysis package (Cox, 1996). The first 5 TRs were excluded from the analysis. Preprocessing for each participant followed a script generated by the AFNI program afni_ proc.py. Voxel time series were aligned to the same temporal origin using the AFNI program 3dTshift and the quintic Lagrange polynomial interpolation option. For each EPI run, each $3 \mathrm{~d}$ volume from the input dataset was registered to the volume acquired in closest temporal proximity to the T1-weighted anatomical scan (the first volume of the first EPI scan) using the AFNI program 3dvolreg with the cubic polynomial interpolation option. A 6-mm full-width half maximum (FWHM) Gaussian blur was then applied using AFNI's 3dmerge program. The data were then scaled in order to calculate the percent signal change. Preprocessed data were analyzed based on the General Linear Model (GLM; Friston, Jezzard, \& Turner, 1994; Josephs, Turner, \& Friston, 1997; Miezin, Maccotta, Ollinger, Petersen, \& Buckner, 2000; Zarahn, Aguirre, \& D'Esposito, 1997), using AFNI's 3dDeconvolve program with the TENT option. The deconvolution analysis estimated the impulse response function (IRF) for each unique condition (LoSemLoSyn vs. HiSemLoSyn vs. LoSemHiSyn vs. HiSemHiSyn), with no assumptions regarding the shape of the function, at the 11 time points (i.e., image acquisitions) immediately following the onset of the first phrase of each sentence, and at the 9 time points immediately following the onset of each comprehension question. The filler trials were modeled separately. The deconvolution analysis produced an IRF for each condition at each voxel. In addition, six motion factors and baseline drifts were also estimated and included in the model. All effects were modeled simultaneously in the GLM for each subject. The structural images were transformed to the Colin template (http://imaging.mrccbu.cam.ac.uk/downloads/Colin/) for each subject using AFNI's @ auto_tlrc program, and then the functional data were aligned using the transform obtained in the previous step.

Because the presentation duration of sentences was longer than questions, the BOLD signal peak post-sentence-onset emerged later and lasted longer than the peak post-question-onset. Therefore, different time points were used to identify BOLD signal peaks for sentences and questions (7.5-15s post sentence onset, $5-7.5 \mathrm{~s}$ post question onset). The intensity values for the BOLD signal peak were averaged for each condition at each voxel, and these values were submitted to an analysis of variance (ANOVA) using AFNI's 3dANOVA program. The filler trials were modeled in the deconvolution analysis, but not included in the ANOVA. The estimated hemodynamic responses were then baseline corrected such that the origin of each IRF was zero. Following the onset of sentences and following the onset of comprehension questions, regions of interference were identified through two voxel-wise contrasts (semantic interference: HiSemLoSyn + HiSemHiSyn vs. LoSemLoSyn + LoSemHiSyn; syntactic interference: LoSemHiSyn+HiSemHiSyn vs. LoSemLoSyn + HiSemLoSyn). Statistical maps were thresholded using a combined significance level of $p<$ 0.01 and cluster size of 70 voxels, resulting in a corrected $p$-value of 0.01 (as determined by the AFNI program 3dClustSim). Then, the estimated hemodynamic response was averaged and extracted from each cluster. The interaction between the effect of interference (semantic 
interference or syntactic interference) and quadric contrast effect of time (across the 11 estimated time points following the onset of sentences and across the 9 estimated time points following the onset of questions) was assessed in each region. Only regions exhibiting a significant interaction effect $(\mathrm{p}<.05)$ and with the maximum BOLD signal at any time point exceeding $|0.2|$ will be reported. These criteria were applied to ensure that the identified regions exhibit reasonable hemodynamic signal changes.

\section{Results}

\subsection{Behavioral Pilot}

First, to examine the two interference effects, reading times of the critical phrase that contained the verb of the main clause and accuracy and response times to comprehension questions were submitted to a two-way repeated measures ANOVA with semantic interference (low vs. high) and syntactic interference (low vs. high) as factors. The means for RT and accuracy by condition are shown in Table 2.

For the reading times of the critical phrase, only trials with accurate verbal responses to the comprehension questions were included. After excluding extreme values $(<200 \mathrm{~ms}$ or $>$ $2000 \mathrm{~ms}$ ), response times $+/-3$ standard deviations from each subject's mean were also excluded from the analysis. The main effect of syntactic interference was significant, $F(1,23)=7.15, p=0.01, M S E=1795$, but not the main effect of semantic interference, $F(1,23)=1.31, p=.26, M S E=1738$, nor the interaction between semantic and syntactic interference, $F(1,23)=.29, p=.60, M S E=2959$. Subjects took longer to read the verb phrases in the high syntactic interference condition (mean $=719 \mathrm{~ms}$ ) than in the low syntactic interference condition (mean $=696 \mathrm{~ms}$ ), replicating Van Dyke (2007) and Van Dyke \& Lewis (2003) who showed that integrating a subject and verb is more difficult when the intervening noun is also a subject.

For the comprehension questions, trials with no responses were counted as errors. Response times $+/-3$ standard deviations from the mean for each subject were excluded from the analysis after excluding the extreme values $(<200 \mathrm{~ms}$ or $>5000 \mathrm{~ms})$. The only significant effect was a semantic interference effect for response times, $F(1,23)=22.26, p<.001, M S E=$ 13698. That is, participants spent longer in responding to questions for sentences in the high semantic interference condition (mean $=1492 \mathrm{~ms}$ ) than in the low semantic interference condition (mean $=1380 \mathrm{~ms}$ ). The semantic interference effect for accuracy was not significant, $F(1,23)=1.15, p=.29, M S E=.001$. In addition, there was no significant main effect of syntactic interference for either response times, $F(1,23)=0.19, p=.26, M S E=$ 1738 , or accuracy, $F(1,23)=1,15, p=.29, M S E=.001$, and no significant interaction between the semantic and syntactic interference for either response times, $F(1,23)=1.13, p$ $=.30, M S E=11769$, or accuracy, $F(1,23)=.60, p=.45, M S E=.002$.

Additionally, reading times of phrases at each serial position of the sentences were analyzed separately for the experimental and filler sentences to determine the phrase presentation durations in the scanner that were the $75^{\text {th }}$ percentile reading times for each phrase in the pilot study. The $75^{\text {th }}$ percentile reading times were determined within each subject at each position (from the first to the last phrase of target sentences: $1343 \mathrm{~ms}, 1271 \mathrm{~ms}, 1624 \mathrm{~ms}$, $1003 \mathrm{~ms}, 896 \mathrm{~ms}$, and $927 \mathrm{~ms}$; from the first to the last phrase of filler sentences: $1360 \mathrm{~ms}$, $1339 \mathrm{~ms}, 1117 \mathrm{~ms}, 1000 \mathrm{~ms}, 1010 \mathrm{~ms}$, and $930 \mathrm{~ms}$ ). To equalize the total presentation durations for all sentences for the fMRI experiment, the $75^{\text {th }}$ percentile reading times of phrases for fillers were adjusted slightly (from the first to the last phrase of filler sentences: $1360 \mathrm{~ms}, 1339 \mathrm{~ms}, 1117 \mathrm{~ms}, 1100 \mathrm{~ms}, 1110 \mathrm{~ms}$, and $1030 \mathrm{~ms}$ ). In addition, after all phrases in each sentence were presented, a fixation cross was presented at the end of each sentence (36 ms for target sentences; $44 \mathrm{~ms}$ for filler sentences). If a sentence only had 5 phrases, a 
fixation was presented as the $6^{\text {th }}$ phrase. Therefore, a total presentation time of $7100 \mathrm{~ms}$ was applied in the fMRI experiment for each sentence.

\section{2 fMRI Behavioral Performance}

In the scanner, phrases were presented at a set rate and thus there were no RT measures for sentence reading. Consequently, only latency and accuracy for the comprehension questions were analyzed. Due to a technical problem with the microphone, response times for three subjects and accuracy data for two subjects were missing. Mean RT and accuracy by condition are shown in Table 2. Two-way repeated measures ANOVAs of semantic interference (low vs. high) and syntactic interference (low vs. high) were conducted for response times of nine subjects and for the accuracy data of ten subjects. For the analysis of accuracy, trials with no responses were counted as errors. Analyses of response times were conducted both for all trials and for trials with accurate verbal responses. As for question answering, the main effects of semantic interference were significant (response times excluding incorrect responses: $F(1,8)=5.80, p<05, M S E=10926$; response times including incorrect responses: $F(1,8)=15.70, p<.01, M S E=10310$; accuracy: $F(1,9)=24.75, p<.001$, $M S E=.006)$. That is, participants spent longer and made more errors in responding to questions for sentences in high semantic interference conditions than low semantic interference conditions. When excluding response times for incorrect responses, the means RTs were 1566 in the low semantic interference condition and 1649 in the high semantic interference condition. When including response times for incorrect responses, these means were 1628 for the low semantic interference condition and 1762 for the high semantic interference condition. The mean accuracies were $80 \%$ in the low semantic interference condition and $68 \%$ in the high semantic interference condition. Also replicating the findings from the pilot experiment for question answering, the main effect of syntactic interference and the interaction effect between the two types of interference were not significant for either accuracy or response times (the syntactic interference effect: response time excluding incorrect responses, $F(1,8)=3.43, p=.10, M S E=63420$; response time including incorrect responses, $F(1,8)=5.18, p=.052, M S E=29657$; accuracy, $F(1,9)=4.79, p=.056, M S E=$. 009; the interaction effect: response time excluding incorrect responses, $F(1,8)=.40, p=.55$, $M S E=16671$; response time including incorrect responses, $F(1,8)=4.66, p=.063, M S E=$ 21411; accuracy, $F(1,9)=4.68, p=.059, M S E=.006)^{2}$.

\subsection{Neuroimaging}

Neuroimaging data for all trials were included in the fMRI analyses. This was motivated by interference theory, which predicts that participants will be distracted by items that are syntactically or semantically similar to the target. Therefore, interference will result in errors or longer response times in retrieving the correct answer, so we were not interested in only successful attempts to resolve interference. Indeed, selection processes aimed at resolving interference are not guaranteed to produce the correct alternative. In fact, the likelihood of an error should be greater in high interference conditions due to the presence of close competitors, which will be selected on a high probability of trials. Hence, "interference resolution," does not necessarily imply that interference has been resolved correctly, only that the participant has made some selection from the competing alternatives. We expect that brain activations in the presence of interference, whether successfully resolved or not, will reflect the greater effort required to process these sentences.

\footnotetext{
${ }^{2}$ We note that several of these effects for question answering involving the syntactic manipulation approached significance. Given low statistical power due to a small N, 95\% confidence intervals on these effects are quite wide (e.g., RTs excluding incorrect responses, $\mathrm{CI}=68.4 \mathrm{~ms} \pm 85.1 \mathrm{~ms}$; accuracy, $\mathrm{CI}=7.0 \% \pm 7.0 \%$ ). Thus we do not have strong grounds for claiming that syntactic effects on question answering were or were not greater than those in the pilot experiment.
} 
3.3.1 Contrasts of interference effects-We conducted two voxel-wise contrasts

( $p_{\text {activation }}<0.01$ with the cluster size $>70$, resulting in $p_{\text {corrected by cluster threshold }}<0.01$ ) on the peak of hemodynamic signal changes following the onset of the sentences $(7.5-15 \mathrm{~s}$ post sentence onset) in order to identify regions exhibiting the effect of semantic interference (HiSemLoSyn+ HiSemHiSyn vs. LoSemLoSyn + LoSemHiSyn) or the effect of syntactic interference (LoSemHiSyn+ HiSemHiSyn vs. LoSemLoSyn + HiSemLoSyn). Moreover, since interference effects were also observed in offline measures of responses to comprehension questions in the study of Van Dyke (2007) and in the behavioral data of this study, the same voxel-wise contrast analyses were also conducted on the peak of hemodynamic signal changes following the onset of questions (5-7.5s post question onset).

We were justified in examining syntactic effects in the neuroimaging data despite the absence of syntactic effects in the behavioral data during the fMRI experiment because the syntactic effects occurred in the reading times only during the pilot experiment. As the fixed phrase-by-phrase presentation in the scanner precluded reading time data, we infer that such effects would nevertheless be present as participants read the sentences in the scanner because the reading task itself was identical aside from the presentation mode.

3.3.1.1 The syntactic interference contrast following the onset of Sentences: See Figure 1 for the activation map. Two regions in the LIFG (centered at $-47,13,16$ : BA44 \& -45 , 26, 9: BA 45; coordinates in this study are all reported in MNI space) were identified that exhibited greater activation for the high syntactic conditions (LoSemHiSyn \& HiSemHiSyn) than the low syntactic conditions (LoSem-LoSyn \& HiSemLoSyn) (see Table 3). The left superior temporal gyrus (STG; centered at $-46,-24,0$ : BA22) (see Table 4) also showed greater activation in the high than low syntactic interference condition. This region is of interest as some researchers have claimed that the region is involved in online syntactic processing (Friederici, Ruschemeyer, Hahne, \& Fiebach, 2003) whereas other have argued that the region is involved only in the retrieval of lexical information (both semantic and syntactic; Snijders et al., 2008; Snijders, Petersson, \& Hagoort, 2010). In addition, several regions in bilateral superior frontal, right medial frontal, inferior frontal and precuneus demonstrated enhanced deactivation for the high vs. low interference conditions (see Appendix B for detailed information). Such regions have often been considered part of a default network that tends to deactivate during task performance.

3.3.1.2 The semantic interference contrast following the onset of Sentences: See Figure 1 for the activation map. No region was identified in this contrast exhibiting greater activation for the high semantic conditions (HiSem-LoSyn \& HiSem-HiSyn) than the low semantic conditions (LoSem-LoSyn \& LoSem-HiSyn). Again, bilateral superior frontal regions showed increased deactivation for the high semantic interference conditions than the low semantic interference conditions. Detailed information about all regions that exhibited differential activation between high vs. low semantic interference conditions in this contrast is listed in Appendix C.

3.3.1.3 The syntactic interference contrast following the onset of Questions: See Figure 2 for the activation map. No region in the LIFG was identified in this contrast exhibiting greater activation for the high syntactic conditions (LoSemHiSyn \& HiSemHiSyn) than the low syntactic conditions (LoSemLoSyn \& HiSemLoSyn). Significant activations for this contrast were observed in the left superior frontal gyrus, the right cuneus, middle occipital gyrus, superior temporal gyrus, and inferior frontal gyrus. Detailed information about all the regions identified in this contrast is listed in Appendix D.

3.3.1.4 The semantic interference contrast following the onset of Questions: See Figure 2 for the activation map. Two regions in the LIFG (centered at $-31,19,-4$ : BA47 \& -50 , 
20, 10: BA 45) (see Table 3) were identified in this contrast exhibiting greater activation for the high semantic conditions (HiSemLoSyn \& HiSemHiSyn) than the low semantic conditions (LoSemLoSyn \& LoSemHiSyn). As for the syntactic contrast during sentence processing, a region in the left STG also showed greater activation in the high than the low semantic conditions (centered at $-45,-27,-27$ : BA 22; see Table 4). Other regions that exhibited this effect were in bilateral superior frontal gyrus, and the left middle frontal gyrus. Detailed information about these regions is listed in Appendix E.

3.3.2 Conjunction analysis of contrasts of interference effects-Since the LIFG and the left STG were identified in both the syntactic interference contrast and the semantic interference contrast (although at different time points, i.e., during sentence reading and during question answering respectively), a conjunction analysis was conducted between the syntactic interference contrast (following the onset of sentences) and the semantic interference contrast (following the onset of comprehension questions). Since regions that are activated in both contrasts are less likely to be noise, a conjunction map results in a smaller false alarm rate than that of each individual contrast. Therefore, a lower value of $p_{\text {corrected by cluster threshold }}<0.05$ ( $p_{\text {activation }}<0.05$ with the cluster size $>243$ ) was applied to each individual contrast that was included in the conjunction analysis.

A region in the LIFG (see Figure 3; centered at BA 45: $-48,24,9$ and extending to BA 44; in a size of 556 voxels) was identified to be activated in both contrasts (the syntactic interference contrast following the onset of sentences \& the semantic interference contrast following the onset of comprehension questions). The left STG regions (see Figure 3; centered at BA22: $-47,-9,-10$; in a size of 69 voxels) that were identified in both contrasts also overlapped with the region involved in the semantic contrast extending to a more posterior area. Interestingly, the BA 47 region that was identified in the semantic interference contrast during participants' response to the comprehension question did not show any overlap with regions exhibiting the syntactic interference effect (see Figure 3). To further test the specific role of BA 47 in semantic interference, an even lower value of $p_{\text {uncorrected }}<0.1$ was applied to each individual contrast that was included in the conjunction analysis. The result showed that the BA 47 region still only exhibited the semantic interference effect.

\section{Discussion}

According to the cue-based parsing theory (Lewis, et al., 2006; Van Dyke, 2007; Van Dyke \& McElree, 2006), when nouns that intervene between a subject and verb possess semantic or syntactic features that partially match the target subject, interference from those distractors produces increased processing difficulty. The aim of the present study was to replicate the behavioral effects of semantic and syntactic interference reported in the study of Van Dyke (2007) and, in addition, to investigate the neural basis involved in managing the two types of interference. We discuss these aims below in turn.

\subsection{Time course of effects}

This study did indeed find the predicted interference effects, although at different stages in processing (sentences vs. questions). Consistent with the findings of Van Dyke (2007), the syntactic interference effect was found in reading times at the critical verb phrase in our pilot experiment. Although the semantic interference effect was not observed in these reading times, it showed up later in response times for comprehension questions. A similar locus of effects was observed in the neuroimaging data: syntactic effects were observed during sentence reading, while semantic effects were observed during questions. 
Van Dyke (2007) reported a similar time course, in which syntactic interference effects appeared in reading times at the critical verb, and semantic effects appeared later, although in the original experiment this effect was also in reading times (at sentence end). Although this time-course difference is consistent with other studies showing semantic effects later than the region containing the anomaly (Boland \& Blodgett, 2001; Braze, Shankweiler, Ni, \& Palumbo, 2002; Ni, Fodor, Crain, \& Shankweiler, 1998), the low temporal resolution of fMRI makes the difference in the current study more difficult to interpret. One possibility is that the semantic interference effect observed during questions was in fact due to a continuation of processing that started during sentence reading but was not complete until past the end of the sentence. This is plausible because the integration of semantic information appears to be slower than the integration of syntactic information; McElree and Griffith $(1995,1998)$ found that violations of syntactic constituent structure were noticed 50-100 ms before violations of theta roles (i.e., semantic fit). This difference in time course is likely due to differences in the unification of syntactic and semantic information. As syntactic processing occurs over a finite set of grammatical features, it will take less time to determine how each noun phrase syntactically matches the critical verb. Moreover, these matching processes are simpler: required syntactic features (i.e., grammatical roles, agreement features, plurality) are either present or not. In contrast, determining the semantic fit between a noun phrase and a verb is more complex due to the possibility of varying degrees of match. For example, consider the experimental sentence "The ambassador who had exposed the known conspiracy during the meeting will arrive" which was considered to have both low semantic and syntactic interference. In this sentence; "conspiracy" will unambiguously be assigned the role of object, which will thus result in a clear non-match on this syntactic feature when the comprehender tries to retrieve the subject of "will arrive." However, although "conspiracy" provides a less good fit semantically to "will arrive" than does "ambassador," there are semantic features of "conspiracy" (such as the fact that a conspiracy implies a group of people) which provide some degree of match to the required semantic features of "will arrive" (i.e., the subject would prototypically be concrete persons or objects). Also, non-concrete subjects often appear with movement verbs when used in a metaphorical sense (e.g., "New Year's will arrive with a bang"). Because of the greater subtleties in determining fit on semantic grounds, the buildup of conflict between the target and distractor on semantic grounds may take a longer time, with the resolution of conflict (and the involvement of the LIFG) coming on line later than is the case in resolving syntactic conflict. Thus, even though such semantic processing may begin immediately upon access to the semantic cues associated with the main verb, conflict resolution processes may not be sufficiently strong to be detectable neurally until a later time point for semantic than syntactic features. It is therefore possible that the semantic interference effect will not appear until after the sentence itself was read, making the observation of this effect during questions unrelated to processing the question itself.

Alternatively, our finding of semantic interference at question answering may suggest that it was only when participant's attention was focused on their error that they invoked processes necessary for distinguishing the target subject from the distractor. This would be consistent with the idea that participants were truly fooled by the semantic distractor, not even noticing the concomitant syntactic anomaly because they have settled on a seemingly plausible interpretation. This would be the outcome of a "Good-Enough" parsing strategy (Christianson, Hollingworth, Halliwell, \& Ferreira, 2001; Ferreira \& Patson, 2007) where participants adopt a low threshold for enforcing syntactic well-formedness during online processing in favor of attainting a semantically plausible interpretation. In this case, the semantic interference effect observed here will be related to a controlled process in which previously processed information is retrieved during offline reanalysis of the incorrect interpretation. 


\subsection{Neuroimaging results: Left Inferior Frontal Gyrus}

Regions in the LIFG were identified in contrasts of both types of interference, with syntactic interference invoking responses centered in BA45 and extending into BA44 and semantic interference invoking responses in BA 45 and BA47. The association of BA 44 and BA 45 and syntactic interference is in line with other findings pointing to a syntactic role for these regions. Although the LIFG (especially Broca's area) has long been considered to be a critical region for syntactic processing (Caplan \& Futter, 1986; Caramazza \& Zurif, 1976; Schwartz, Saffran, \& Marin, 1980), recent insights from neuropsychological and neuroimaging research have led to alternative interpretations of its function (e.g., Badre \& Wagner, 2005; Caplan, Hildebrandt, \& Makris, 1996; Jonides, Smith, Marshuetz, \& Koeppe, 1998; Kan \& Thompson-Schill, 2004a; Thompson-Schill et al., 1997). In response to these findings, some researchers have argued that the LIFG is not an area involved in syntactic processing per se, but rather is recruited when the demand for working memory is high in comprehension tasks (Caplan \& Waters, 1999; (Fiebach, Schlesewsk, Lohmann, Cramon, \& Friederici, 2005; Waters \& Caplan, 2005). However, the current results suggest that the role of the LIFG in sentence comprehension cannot be related simply to storing unintegrated information in working memory because the high and low semantic interference conditions in the present study have the same syntactic structure and the same number of unintegrated noun phrases. Thus, demands for working memory capacity should be equated in these conditions. Consequently, our findings would argue against the claim that involvement of the LIFG in sentence processing necessarily results from working memory demands (Fiebach et al., 2005; Makuuchi, Bahlmann, Anwander, \& Friederici, 2009).

Rather, our results are more consistent with those who emphasize the role of LIFG in cognitive control (Hagoort, 2005; 2009; Novick et al., 2009; Novick et al., 2005; Ye \& Zhou, 2008, 2009a, 2009b), as our syntactic and semantic manipulations served to increase the number of distractors for identifying the desired subject noun along each dimension. Accordingly, our conjunction analysis revealed that a common area restricted to BA45 was associated with both types of interference. This result is consistent with the view that this area in particular is involved in post-retrieval selection among representations retrieved via automatic cue-based associative retrieval (Badre et al., 2005; Badre \& Wagner, 2007; Kan \& Thompson-Schill, 2004a; Moss et al., 2005; Thompson-Schill, et al., 1997; Thothathiri, Kim, Trueswell, \& Thompson-Schill, 2012).

Our observation that a region in BA 47 was involved only in semantic interference during questions is consistent with the idea that there may be somewhat different regions within LIFG specific to semantic and syntactic interference. However interpretation of function underlying this result is confounded by the uncertainty discussed above, related to whether the semantic interference effect appears online during reading, or offline during question answering. If this is an online effect, then our results may be consistent with the unification model of Hagoort and colleagues (Hagoort, 2005; 2009; Snijders et al., 2008), who suggested that BA 47 and BA 45 are involved in semantic processing, while BA 45 and BA 44 are involved in syntactic processing. Additionally, in a study examining sentence sequencing, Bornkessel-Schlesewsky, Grewe, and Schlesewsky (2012) proposed a similar idea that the anterior-superior portion of LIFG contributed to linking the semantic/pragmatic information of the current sentence with the broader discourse, whereas the posteriorinferior LIFG is involved in processing argument prominence.

Alternatively, if our semantic interference effect is associated with an offline process, then our results are consistent with the view of Badre and colleagues (Badre et al., 2005; Badre \& Wagner, 2007; Wagner, Paré-Blagoev, Clark, \& Poldrack, 2001), who argued that BA47 in particular is associated with a controlled retrieval process which interacts with the stored semantic knowledge in lateral temporal cortex and is distinct from the selection process 
associated with BA45. We prefer this interpretation on purely theoretical grounds, due to specifics of the cue-based parsing model, which differ from the unification approach.

Specifically, the Hagoort model suggests that LIFG is important for unification because of its role in maintaining representations retrieved from posterior temporal regions. In contrast, the cue-based parsing approach deemphasizes the need for maintaining active representations, in favor of a fast cue-based retrieval mechanism that restores representations into the focus of attention as needed (Lewis et al., 2006). On this view, the inappropriate activation of similar distractors is inevitable when cues provide partial matches to non-target information, making the selection aspect of cognitive control, and not the maintenance aspect, more relevant for explaining interference effects. In terms of the Badre model, the conflict produced by the semantic distractors would produce the activations in the putative post-retrieval selection region (BA45) observed here, while the need to deliberately revise an incorrect semantic interpretation would produce activations in the region that supports controlled retrieval (B A47) such as those observed here.

\subsection{The left mid-STG}

In addition to the regions in the LIFG, a small portion of the left mid-STG was also activated in both syntactic and semantic interference contrasts, with the activation associated with the semantic interference effect extending to a more posterior area. These findings are in line with a number of other reports of the involvement of regions of the superior temporal gyrus in semantic and syntactic processing (Caplan, Alpert, \& Waters, 1998; Caplan, Stanczak, \& Waters, 2008; Fiebach et al., 2004; Friederici et al., 2003; Just et al., 1996; Rodd et al., 2010). In particular, Friederici and colleagues (2003) found that the mid and posterior portion of the STG was more activated for sentences with semantic violations than for correct sentences. In contrast, the anterior portion of the STG was more engaged for sentences with syntactic violations than correct sentences. The dissociated neural basis of semantic and syntactic processing is consistent with the activation pattern observed in the present study with the semantic region posterior to the syntactic region. Interestingly, however, our results show that these regions are involved in the processing of sentences which do not contain violations but instead differ in their degree of semantic or syntactic interference.

The association of the these regions with our syntactic and semantic interference effects suggests that, contrary to the view of Hagoort (2005) which argues that retrieval is the only role of the posterior superior temporal gyrus, integration of syntactic and semantic information occurs in posterior temporal cortex. As discussed in the introduction, retrieval interference occurs as a side effect of a global matching process, guided by a retrieval probe derived from syntactic and semantic cues associated with particular lexical items and the current state of the parse. Hence, a pre-requisite for interference effects is the integration of syntactic and semantic cues to create the retrieval probe. Friederici (2012) suggested that this integration occurs in the posterior temporal cortex (Bornkessel Zysset, Friederici, Von Cramon, \& Schlesewsky, M., 2005; Friederici, 2011; Newman, Ikuta, \& Burns, 2010), though she was not referring to the integration of semantic and syntactic retrieval cues, per se, but rather to different types of linguistic knowledge.

Positioning feature integration in STG regions is also consistent with findings that suggest syntactic and semantic integration can occur without involvement of the LIFG when conflict is not present. For instance, patients with LIFG damage can show good comprehension of sentences with low conflict (Novick et al., 2009) and agrammatic aphasic patients (who are assumed to have LIFG damage) tend to do well on grammaticality judgments (Linebarger et al., 1983). Moreover, some fMRI studies have shown an absence of activation in frontal regions for the comprehension of coherent passages where conflict between representations is presumably minimized (e.g., Brennan et al., 2012). Thus, we would suggest that the 
retrieval of semantic and syntactic features of words, the generation of retrieval cues, and the integration of representations based on a match between retrieval cues and features of encoding representations may all occur without involvement of the LIFG. Greater activation in the STG in the high interference conditions may result because more representations are retrieved due to the partial match with retrieval cues. The LIFG comes on line when there is a need to resolve this interference generated by similar competitors in the linguistic context.

\subsection{Other regions}

The superior frontal region (BA 9/10) was activated in both interference contrasts following the onset of comprehension questions, but not identified in the contrasts following the onset of sentences (although it showed greater deactivation in high vs. low interference conditions during sentence processing). This area may be involved in the post-interpretive processing proposed by Caplan and Waters (1998), in which readers use propositional content of the sentences to accomplish additional tasks, such as answering the comprehension questions in this study. Moreover, there were regions in the medial frontal, superior frontal, prefrontal and precuneus exhibiting enhanced deactivations for high vs. low interference conditions following the onset of sentences and comprehension questions. These regions have often been considered part of a "default mode network" that shows greater deactivation when more cognitive resources are occupied by the ongoing task (e.g., Bluhm et al., 2010; Buckner \& Vincent, 2007; Mason et al., 2007).

\subsection{Inclusion of inaccurate trials}

We included both accurate and inaccurate trials in the neuroimaging analyses because we assumed that subjects attempted to resolve interference on inaccurate trials but were unsuccessful in doing so $^{3}$. In understanding this approach, it is necessary to realize that our comprehension questions were unlike those employed in most experiments, in which the question is present primarily to prevent attention lapses, and often appears on only a subset of trials. In these studies, the content of the question is usually incidental to the critical manipulation, querying general aspects of the read material so that they are easy to answer correctly. Due to this, accuracy levels are typically near $100 \%$ and do not raise an issue for data analysis (e.g., Bornkessel et al, 2005; Mason, et al., 2003; Makuuchi, et al., 2009; Thothathiri et al., 2012). In the present experiment, the comprehension questions were designed specifically to assess individuals' identification of the subject of the critical verb (i.e., the manipulated dependency) and could therefore reveal susceptibility to interference from the distracting noun. This made the comprehension questions critical data in their own right, with the theoretically motivated prediction that significantly lower accuracy levels will be found in conditions with high interference precisely because the incorrect noun phrase is retrieved. This is both a direct and necessary consequence of a cue-based retrieval mechanism that utilizes global matching processes. Therefore, the greater activations we found in the LIFG and the mid-STG regions for high interference conditions than low interference conditions are more likely due to the influence of the distractor, and not to simple lapses in attention. Moreover, the inclusion of comprehension questions on every trial, regardless of the amount of interference, discouraged attention lapses in general, and meant that any incidental lapses would likely be evenly distributed across conditions, rather than focused on any particular condition in the experiment.

A possible criticism of this approach is that the inclusion of incorrect trials may increase noise in our dataset because of possible error detection processes. We note, however, that the region responsible for error detection has been found to be the anterior cingulate cortex

\footnotetext{
${ }^{3}$ This assumption is supported by the findings that participants spent longer time in responding to high semantic interference sentences than low semantic interference sentences regardless of whether inaccurate trials were included in the analyses.
} 
(e.g., Carter, Braver, Botvinich, Noll, \& Cohen, 1998; Yeung, Botvinich, \& Cohen, 2004 ; Van Veen \& Carter, 2002), and not the regions we identified in the study.

\section{Conclusion}

The present study replicated behavioral findings pointing to a cue-based retrieval mechanism as the means for creating dependencies between non-adjacent linguistic constituents. With regard to the neural basis of these processes, regions in the LIFG were activated during interference with BA 44 and 45 showing greater activation in the presence of increased syntactic interference and BA 45 and 47 showing greater activation in the presence of increased semantic interference. A conjunction analysis of activations in both types of interference revealed a region centered in BA45 and extending into BA44 which was associated with both syntactic and semantic interference. The region of semantic interference in BA 47, however, did not overlap with any region of syntactic interference. Thus, there appear to be overlapping but distinct regions within LIFG that mediate semantic and syntactic interference. Importantly, the behavioral and imaging results could not be attributed to greater working memory capacity demands for the high versus low interference conditions. The results instead support a direct role for these regions in resolving retrieval interference, with the region in BA45 associated with post-retrieval selection and the region in BA47 associated with controlled retrieval of semantic information.

\section{Supplementary Material}

Refer to Web version on PubMed Central for supplementary material.

\section{Acknowledgments}

This research was supported by the Gertrude Maurin Fund for Cognitive Psychology at Rice University, by funding for the Human Neuroimaging Lab at Baylor College of Medicine, and by the following grants from the NIH National Institute of Child Health and Human Development: HD 058944 and HD 073288 to Julie Van Dyke (PI), HD 056200 to Brian McElree (PI). The authors would like to thank Vips Patel for assistance in fMRI data collection and Mark Plitt for assistance in coding the verbal responses.

\section{References}

Badre D, Poldrack RA, Paré-Blagoev EJ, Insler RZ, Wagner AD. Dissociable controlled retrieval and generalized selection mechanisms in ventrolateral prefrontal cortex. Neuron. 2005; 47:907. [PubMed: 16157284]

Badre D, Wagner AD. Semantic retrieval, mnemonic control, and prefrontal cortex. Behavioral and Cognitive Neuroscience Reviews. 2002; 1:206-218. [PubMed: 17715593]

Badre D, Wagner AD. Frontal lobe mechanisms that resolve proactive interference. Cerebral Cortex. 2005; 15:2003-2012. [PubMed: 15788702]

Badre D, Wagner AD. Left ventrolateral prefrontal cortex and the cognitive control of memory. Neuropsychologia. 2007; 45:2883-2901. [PubMed: 17675110]

Bluhm RL, Clark R, McFariane AC, Moores KA, Shaw ME, Lanius RA. Default network connectivity during a working memory task. Human Brain Mapping. 2010; 32:1029-1035. [PubMed: 20648663]

Boland JE, Blodgett A. Understanding the constraints on syntactic generation: Lexical bias and discourse congruency effects on eye movements. Journal of Memory and Language. 2001; 45:381411.

Bornkessel-Schlesewsky I, Grewe T, Schlesewsky M. Prominence vs. aboutness in sequencing: a functional distinction within the left inferior frontal gyrus. Brain \& Language. 2012; 120:96-107. [PubMed: 20655580]

Bornkessel I, Zysset S, Friederici AD, Von Cramon DY, Schlesewsky M. Who did what to whom? The neural basis of argument hierarchies during language comprehension. NeuroImage. 2005; 26:221-233. [PubMed: 15862222] 
Braver TS, Cohen JD, Nystrom LE, Jonides J, Smith EE, Noll DC. A parametric study of prefrontal cortex involvement in human working memory. NeuroImage. 1997; 5:49-62. [PubMed: 9038284]

Braze D, Shankweiler D, Ni W, Palumbo L. Readers' eye movements distinguish anomalies of form and content. Journal of Psycholinguistic Research. 2002; 31:25-44. [PubMed: 11924838]

Brennan J, Nir Y, Hasson U, Malach R, Heeger DJ, Pylkkänen L. Syntactic structure building in the anterior temporal lobe during natural story listening. Brain and language. 2012; 120:163-173. [PubMed: 20472279]

Buckner RL, Vincent JL. Unrest at rest: default activity and spontaneous network correlations. NeuroImage. 2007; 37:1091-1096. [PubMed: 17368915]

Caplan D, Alpert N, Waters GS. Effects of syntactic structure and propositional number on patterns of regional cerebral blood flow. Journal of Cognitive Neuroscience. 1998; 10:541-552. [PubMed: 9712683]

Caplan D, Futter C. Assignment of thematic roles to nouns in sentence comprehension by an agrammatic patient. Brain and Language. 1986; 27:117-134. [PubMed: 3947937]

Caplan D, Hildebrandt N, Makris N. Location of lesions in stroke patients with deficits in syntactic processing in sentence comprehension. Brain. 1996; 119:933-949. [PubMed: 8673503]

Caplan D, Stanczak L, Waters G. Syntactic and thematic constraint effects on blood oxygenation level dependent signal correlates of comprehension of relative clauses. Journal of Cognitive Neuroscience. 2008; 20:643-656. [PubMed: 18052788]

Caplan D, Waters GS. Verbal working memory and sentence comprehension. Behavioral \& Brain Science. 1999; 22:114-126.

Caramazza A, Zurif EB. Dissociation of algorithmic and heuristic processes in language comprehension: Evidence from aphasia. Brain and Language. 1976; 3:572-582. [PubMed: 974731]

Carter CS, Braver TS, Barch DM, Botvinick MM, Cohen JD. Anterior cingulate cortex, error detection, and online monitoring of performance. Science. 1998; 280:747-749. [PubMed: 9563953]

Christianson K, Hollingworth A, Halliwell JF, Ferreira F. Thematic roles assigned along the garden path linger. Cognitive psychology. 2001; 42:368-407. [PubMed: 11368528]

Clark SE, Gronlund SD. Global matching models of recognition memory: How the models match the data. Psychonomic Bulletin \& Review. 1996; 3:37-60.

Cohen JD, MacWhinney B, Flatt M, Provost J. PsyScope: a new graphic interactive environment for designing psychology experiments. Behavioral Research Methods, Instruments, and Computers. 1993; 25:257-271.

Cowan N. The magical number 4 in short-term memory: a reconsideration of mental storage capacity. Behavioral and Brain Sciences. 2001; 24:87-114. [PubMed: 11515286]

Cox RW. AFNI: Software for analysis and visualization of functional magnetic resonance neuroimages. Computers and Biomedical Research. 1996; 29:162-173. [PubMed: 8812068]

Ferreira F, Patson ND. The 'good enough' approach to language comprehension. Language and Linguistics Compass. 2007; 1:71-83.

Fiebach CJ, Schlesewsky M, Lohmann G, von Cramon DY, Friederici AD. Revisiting the role of Broca's area in sentence processing: syntactic integration versus syntactic working memory. Human Brain Mapping. 2005; 24:79-91. [PubMed: 15455462]

Fiebach J, Vos SH, Friederici AD. Neural correlates of syntactic ambiguity in sentence comprehension for low and high span readers. Journal of Cognitive Neuroscience. 2004; 16:1562-1575. [PubMed: 15601519]

Fletcher PC, Shallice T, Dolan RJ. "Sculpting the response space" —an account of left prefrontal activation at encoding. NeuroImage. 2000; 12:404-417. [PubMed: 10988034]

Friederici AD. The brain basis of language processing: from structure to function. Physiological reviews. 2011; 91:1357-1392. [PubMed: 22013214]

Friederici AD. The cortical language circuit: from auditory perception to sentence comprehension. Trends in cognitive sciences. 2012; 16:262-268. [PubMed: 22516238] 
Friederici AD, Ruschemeyer S, Hahne A, Fiebach CJ. The role of left inferior frontal and superior temporal cortex in sentence comprehension: localizing syntactic and semantic processes. Cerebral Cortex. 2003; 13:170-177. [PubMed: 12507948]

Friston KJ, Jezzard P, Turner R. Analysis of functional MRI time-series. Human Brain Mapping. 1994; 1:153-171.

Gibson, E. The dependency locality theory: A distance-based theory of linguistic complexity. In: Marantz, A., editor. Image, language, brain: Papers from the first Mind Articulation Project symposium. Cambridge, MA: MIT Press; 2000. p. 94-126.

Gillund G, Shiffrin RM. A retrieval model for both recognition and recall. Psychological Review. 1984; 91:1-67. [PubMed: 6571421]

Hagoort P. On broca, brain, and binding: a new framework. Trends in Cognitive Science. 2005; 9:416423.

Hagoort, P. Reflections on the neurobiology of syntax. In: Bickerton, D.; Szathmáry, E., editors. Biological foundations and origin of syntax. Cambridge, MA: MIT Press; 2009. p. 279-296.

Hamilton AC, Martin RC. Dissociations among tasks involving inhibition: a single-case study. Cognitive, affective, \& Behavioral Neuroscience. 2005; 5:1-13.

Hintzman DL. Judgments of frequency and recognition memory in a multiple-trace memory model. Psychological Review. 1988; 95:528-551.

Howard D, Nickels L, Coltheart M, Cole-Virtue J. Cumulative semantic inhibition in picture naming: experimental and computational studies. Cognition. 2006; 100:464-482. [PubMed: 16413014]

Humphreys MS, Bain JD, Pike R. Different ways to cue a coherent memory system: a theory for episodic, semantic, and procedural tasks. Psychological Review. 1989; 96:208-233.

January D, Trueswell JC, Thompson-Schill SL. Co-localization of Stroop and syntactic ambiguity resolution in Broca's area: implications for the neural basis of sentence processing. Journal of Cognitive Neuroscience. 2009; 21:2434-2444. [PubMed: 19199402]

Jonides J, Smith EE, Marshuetz C, Koeppe RA. Inhibition in verbal working memory revealed by brain activation. Proceedings of the National Academy of Sciences. 1998; 95:8410-8413.

Josephs O, Turner R, Friston KJ. Event-related fMRI. Human Brain Mapping. 1997; 5:243-248. [PubMed: 20408223]

Just MA, Carpenter PA. A capacity theory of comprehension: individual differences in working memory. Psychological Review. 1992; 99:122-149. [PubMed: 1546114]

Just MA, Carpenter PA, Keller TA, Eddy WF, Thulborn KR. Brain activation modulated by sentence comprehension. Science. 1996; 274:114-116. [PubMed: 8810246]

Kan IP, Thompson-Schill SL. Effect of name agreement on prefrontal activity during overt and covert picture naming. Cognitive, Affective, \& Behavioral Neuroscience. 2004a; 4:43-57.

Kan IP, Thompson-Schill SL. Selection from perceptual and conceptual representations. Cognitive, affective, \& Behavioral Neuroscience. 2004b; 4:466-482.

Lewis RL, Vasishth S. An activation-based model of sentence processing as skilled memory retrieval. Cognitive Science. 2005; 29:375-419. [PubMed: 21702779]

Lewis RL, Vasishth S, Van Dyke JA. Computational principles of working memory in sentence comprehension. Trends in Cognitive Sciences. 2006; 10:447-454. [PubMed: 16949330]

Linebarger MC, Schwartz MF, Saffran EM. Sensitivity to grammatical structure in so-called agrammatic aphasics. Cognition. 1983; 13:361-392. [PubMed: 6683142]

Makuuchi M, Bahlmann J, Anwander A, Friederici AD. Segregating the core computational faculty of human language from working memory. Proceedings of the National Academy of Sciences. 2009; 106:8362-8367.

Mason RA, Just MA, Keller TA, Carpenter PA. Ambiguity in the brain: What brain imaging reveals about the processing of syntactically ambiguous sentences. Journal of Experimental Psychology: Learning, Memory, \& Cognition. 2003; 29:1319-1338.

Mason MF, Norton MI, Van Hom JD, Wegner DM, Grafton ST, Macrae CN. Wandering minds: the default network and stimulus-independent thought. Science. 2007; 315:393-395. [PubMed: 17234951] 
McElree, B. Accessing recent events. In: Ross, BH., editor. The Psychology of Learning and Motivation. Vol. 46. Academic Press; 2006.

McElree B, Foraker S, Dyer L. Memory structures that subserve sentence comprehension. Journal of Memory and Language. 2003; 48:67-91.

McElree B, Griffith T. Syntactic and thematic processing in sentence comprehension: Evidence for a temporal dissociation. Journal of Experimental Psychology: Learning Memory, and Cognition. $1995 ; 21: 134-157$.

McElree B, Griffith T. Structural and lexical constraints on filling gaps during sentence processing: A time-course analysis. Journal of Experimental Psychology: Learning, Memory, and Cognition. 1998; 24:432-460.

Miezin FM, Maccotta L, Ollinger JM, Petersen SE, Buckner RL. Characterizing the hemodynamic response: Effects of presentation rate, sampling procedure, and the possibility of ordering brain activity based on relative timing. NeuroImage. 2000; 11:735-759. [PubMed: 10860799]

Moss HE, Abdallah S, Fletcher P, Bright P, Pilgrim L, Acres K, Tyler LK. Selecting among competing alternatives: Selection and retrieval in the left inferior frontal gyrus. Cerebral Cortex. 2005; 15:1723-1735. [PubMed: 15728742]

Murdock BB. A theory of the storage and retrieval of item and associative information. Psychological Review. 1982; 89:609-626.

Newman SD, Ikuta T, Burns T Jr. The effect of semantic relatedness on syntactic analysis: an fMRI study. Brain and language. 2010; 113:51-58. [PubMed: 20304477]

Ni W, Fodor JD, Crain S, Shankweiler D. Anomaly detection: Eye movement patterns. Journal of Psycholinguistic Research. 1998; 27:515-539. [PubMed: 9750312]

Ni W, Constable RT, Mencl WE, Pugh KR, Fulbright RK, Shaywitz SE, Shankweiler D. An eventrelated neuroimaging study distinguishing form and content in sentence processing. Journal of Cognitive Neuroscience. 2000; 12:120-133. [PubMed: 10769310]

Norman KA, O'Reilly RC. Modeling hippocampal and neocortical contributions to recognition memory: a complementary learning systems approach. Psychological Review. 2003; 110:611-646. [PubMed: 14599236]

Novick JM, Trueswell JC, Thompson-Schill SL. Cognitive control and parsing: reexamining the role of Broca's area in sentence comprehension. Cognitive, Affective, \& Behavioral Neuroscience. 2005; 5:263-281.

Novick JM, Kan IP, Trueswell JC, Thompson-Schill SL. A case for conflict across multiple domains: memory and language impairments following damage to ventrolateral prefrontal cortex. Cognitive Neuropsychology. 2009; 26:527-567. [PubMed: 20183014]

Oberauer K. Access to information in working memory: exploring the focus of attention. Journal of Experimental Psychology: learning, Memory, and Cognition. 2002; 28:411-421.

Oppenheim GM, Dell GS, Schwartz MF. The dark side of incremental learning: A model of cumulative semantic interference during lexical access in speech production. Cognition. 2010; 114:227-252. [PubMed: 19854436]

Rodd JM, Longe OA, Randall B, Tyler LK. The functional organization of the fronto-temporal language system: evidence from syntactic and semantic ambiguity. Neuropsychologia. 2010; 48:1324-1335. [PubMed: 20038434]

Schnur TT, Schwartz MF, Kimberg DY, Hirshorn E, Coslett HB, Thompson-Schill SL. Localizing interference during naming: Convergent neuroimaging and neuropsychological evidence for the function of Broca's area. Proceedings of the National Academy of Sciences. 2009; 106:322-327.

Schwartz MF, Saffran E, Marin OSM. The word order problem in agrammatism. I. Comprehension. Brain and Language. 1980; 10:249-262. [PubMed: 7407546]

Shiffrin RM, Steyvers M. A model of recognition memory: REM — retrieving effectively from memory. Psychological Bulletin and Review. 1997; 4:145-166.

Snijders TM, Petersson KM, Hagoort P. Effective connectivity of cortical and subcortical regions during unification of sentence structure. NeuroImage. 2010; 52:1633-1644. [PubMed: 20493954]

Snijders TM, Vosse T, Kempen G, Van Berkum JJA, Petersson KM, Hagoort P. Retrieval and unification of syntactic structure in sentence comprehension: an fMRI study using word-category ambiguity. Cerebral Cortex. 2008; 52:1633-1644. 
Tabor W, Hutchins S. Evidence for self-organized sentence processing: digging-in effects. Journal of Experimental Psychology: Learning, Memory, and Cognition. 2004; 30:431.

Thompson-Schill SL, D'Esposito M, Aguirre GK, Farah MJ. Role of left inferior prefrontal cortex in retrieval of semantic knowledge: A reevaluation. Proceedings of the National Academy of Sciences. 1997; 94:14792-14797.

Thothathiri M, Kim A, Trueswell JC, Thompson-Schill SL. Parametric effects of syntactic-semantic conflict in Broca's area during sentence processing. Brain and language. 2012; 120:259-264. [PubMed: 22225974]

Tomita H, Ohbayashi M, Nakahara K, Hasegawa I, Miyashita Y. Top-down signal from prefrontal cortex in executive control of memory retrieval. Nature. 1999; 401:699-703. [PubMed: 10537108]

Van Dyke JA. Interference effects from grammatically unavailable constituents sentence processing. Journal of Experimental Psychology: Learning, Memory, and Cognition. 2007; 33:407-430.

Van Dyke JA, Johns CL. Memory Interference as a Determinant of Language Comprehension. Language and Linguistics Compass. 2012; 6(4):193-211. [PubMed: 22773927]

Van Dyke JA, Lewis RL. Distinguishing effects of structure and decay on attachment and repair: A retrieval interference theory of recovery from misanalyzed ambiguities. Journal of Memory and Language. 2003; 49:285-413.

Van Dyke JA, McElree B. Retrieval interference in sentence comprehension. Journal of Memory and Language. 2006; 55:157-166. [PubMed: 18209744]

Van Dyke JA, McElree B. Cue-dependent interference in comprehension. Journal of Memory and Language. 2011; 65:247-263. [PubMed: 21927535]

Van Veen V, Carter CS. The anterior cingulate as a conflict monitor: fMRI and ERP studies. Physiology \& Behavior. 2002; 77:477-482. [PubMed: 12526986]

Vosse T, Kempen G. Syntactic structure assembly in human parsing: A computational model based on competitive inhibition and a lexicalist grammar. Cognition. 2000; 75:105-143. [PubMed: 10771275]

Vuong LC, Martin RC. LIFG-based attentional control and the resolution of lexical ambiguities in sentence context. Brain and Language. 2011; 116:22-32. [PubMed: 20971500]

Wagner AD, Paré-Blagoev EJ, Clark J, Poldrack RA. Recovering meaning: left prefrontal cortex guides controlled semantic retrieval. Neuron. 2001; 31:329-338. [PubMed: 11502262]

Waters GS, Caplan D. The relationship between age, processing speed, working memory capacity and language comprehension. Memory. 2005; 13:403-413. [PubMed: 15952262]

Ye Z, Zhou X. Involvement of cognitive control in sentence comprehension: evidence from ERPs. Brain Research. 2008; 1203:103-115. [PubMed: 18313650]

Ye Z, Zhou X. Conflict control during sentence comprehension: fMRI evidence. NeuroImage. 2009a; 48:280-290. [PubMed: 19540923]

Ye Z, Zhou X. Executive control in language processing. Neuroscience and Biobehavioral Reviews. 2009b; 33:1168-1177. [PubMed: 19747595]

Yeung N, Botvinich MM, Cohen JD. The neural basis of error detection: conflict monitoring and the error-related negativity. Psychological Review. 2004; 111:931-959. [PubMed: 15482068]

Zarahn E, Aguirre GK, D'Esposito M. Empirical analyses of BOLD fMRI statistics. I. Spatially unsmoothed data collected under null-hypothesis conditions. NeuroImage. 1997; 5:179-197. [PubMed: 9345548]

Zhang JX, Feng CM, Fox PT, Gao JH, Tan LH. Is left inferior frontal gyrus a general mechanism for selection? NeuroImage. 2004; 23:596-603. [PubMed: 15488409] 
- The neural basis of interference resolution in sentence comprehension was examined.

- Syntactic and semantic interference were observed, but with a different time course.

- $\quad$ BA 45 and the superior temporal gyrus were sensitive to both types of interference.

- A region in BA 47 responded specifically to semantic interference resolution.

- These interference effects could not be due to differential working memory demands. 
Syntactic Interference Contrast

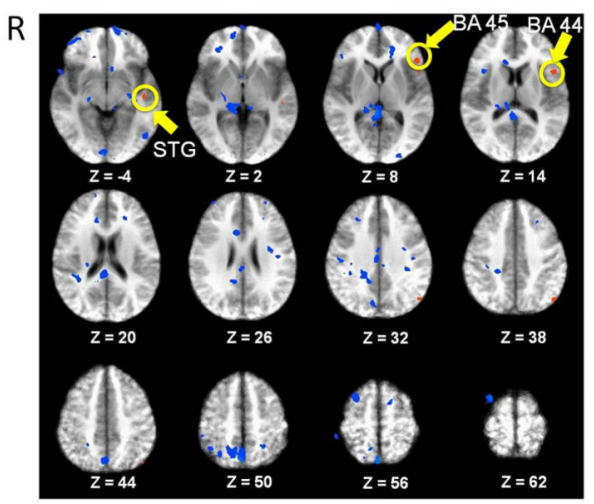

Semantic Interference Contrast

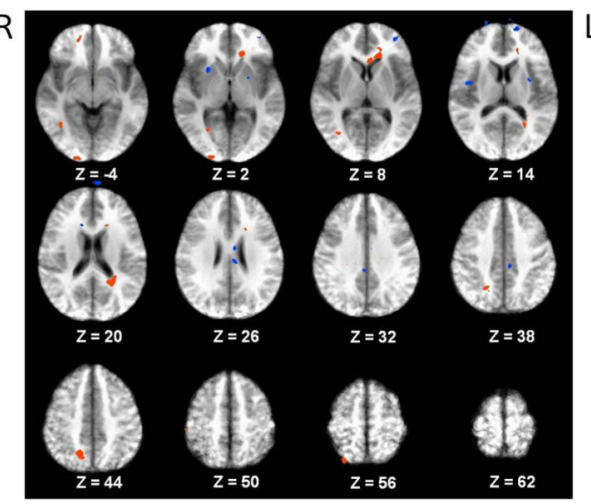

Figure 1.

The results of the contrasts based on signals immediately following the onset of sentences ( $p_{\text {corrected by cluster threshold }}<0.01$ ). STG standards for superior temporal gyrus. The red color represents the regions exhibiting greater activation for the high interference conditions compared to the low interference conditions. The blue color the regions exhibiting greater activation for the low interference conditions compared to the high interference conditions or the regions exhibiting greater deactivation for the high interference conditions compared to the low interference conditions. 
Syntactic Interference Contrast

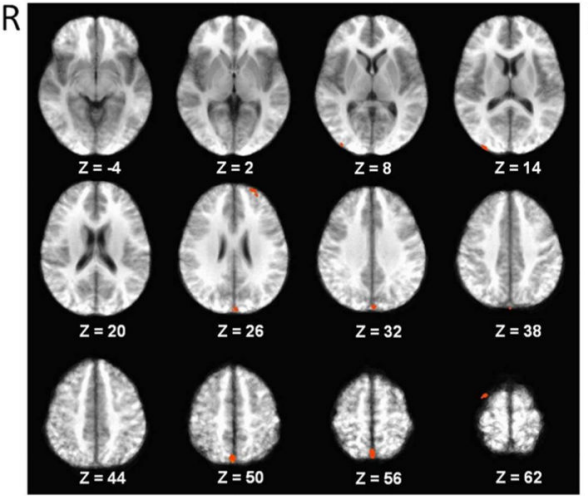

Semantic Interference Contrast

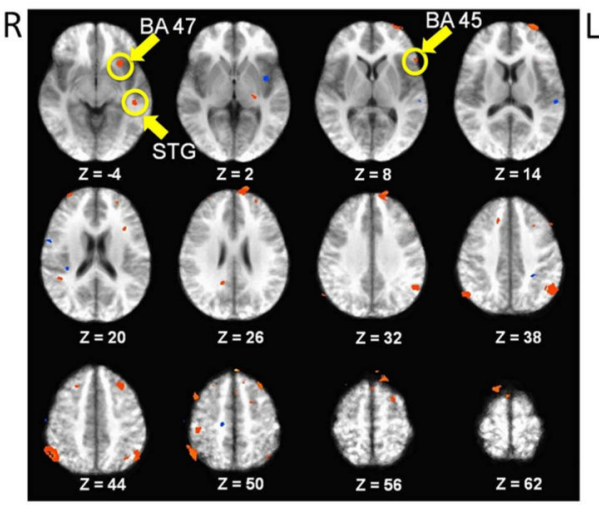

Figure 2.

The results of the contrasts based on signals immediately following the onset of questions $\left(p_{\text {corrected by cluster threshold }}<0.01\right)$. STG standards for superior temporal gyrus. The red color represents the regions exhibiting greater activation for the high interference conditions compared to the low interference conditions or the regions exhibiting greater deactivation for the low interference conditions compared to the high interference conditions. The blue color the regions exhibiting greater activation for the low interference conditions compared to the high interference conditions or the regions exhibiting greater deactivation for the high interference conditions compared to the low interference conditions. 


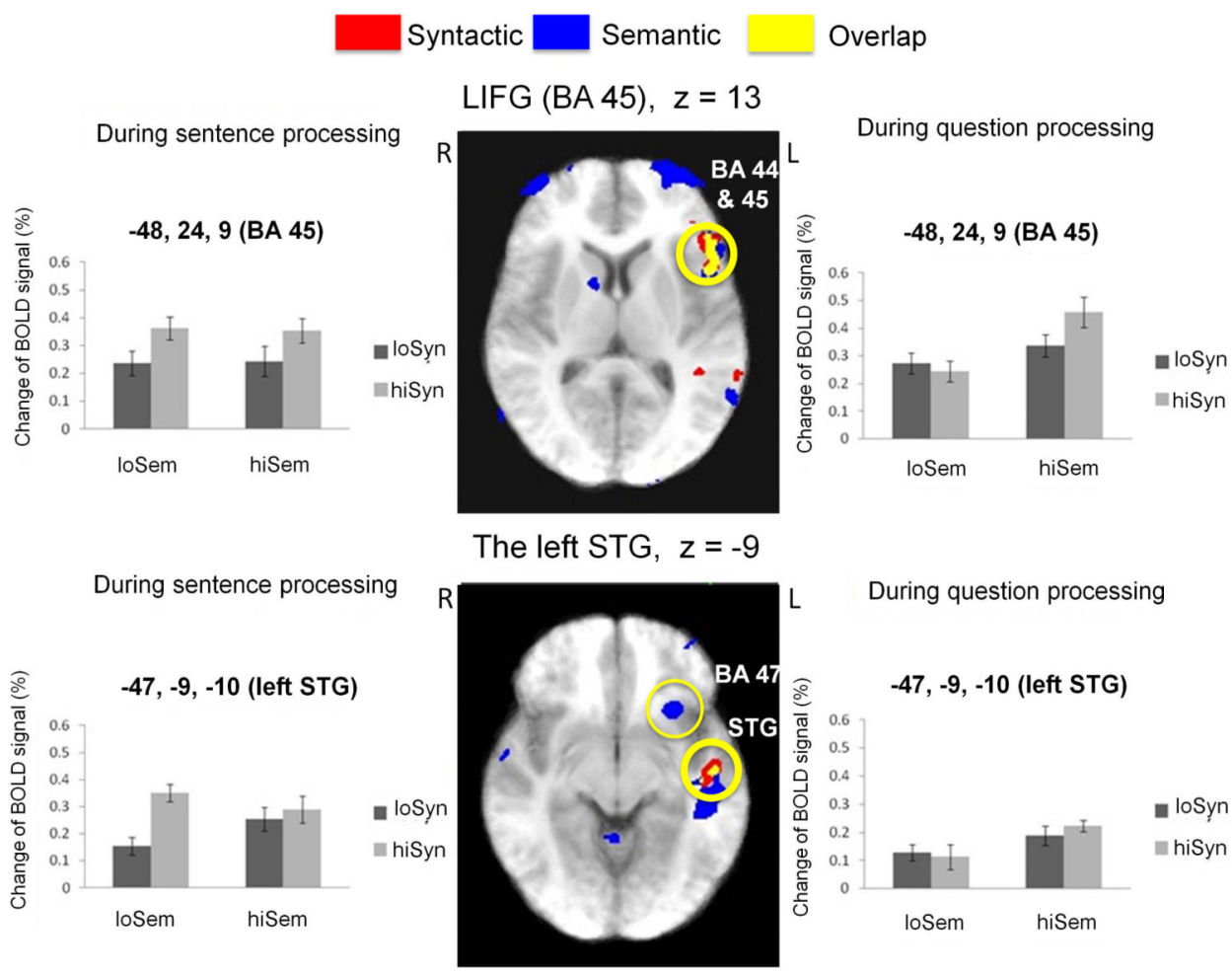

Figure 3.

The results of the conjunction analysis between the syntactic interference contrast following onset of sentences and the semantic interference contrast following onset of comprehension questions (the red color represents the regions exhibiting the syntactic interference effect; the blue color represents the regions exhibiting the semantic interference effect; the yellow color represents the overlapped regions; $\left.p_{\text {corrected by cluster threshold }}<0.05\right)$, and the BOLD signal changes for each interference condition in each overlapped region ("loSyn" represents the low syntactic interference conditions; "hiSyn" represents the high syntactic interference conditions; "loSem" represents the low semantic interference conditions; "hiSem" represents the high semantic interference conditions). Error bars denote standard errors corrected for between-subject variability. 


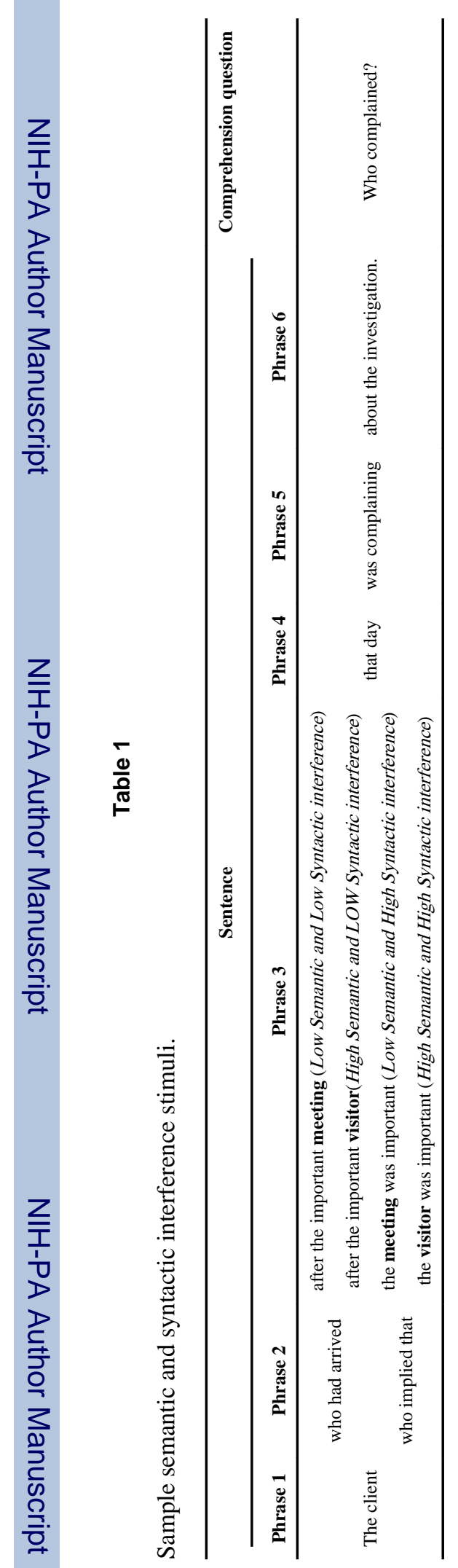

Brain Lang. Author manuscript; available in PMC 2014 September 01. 


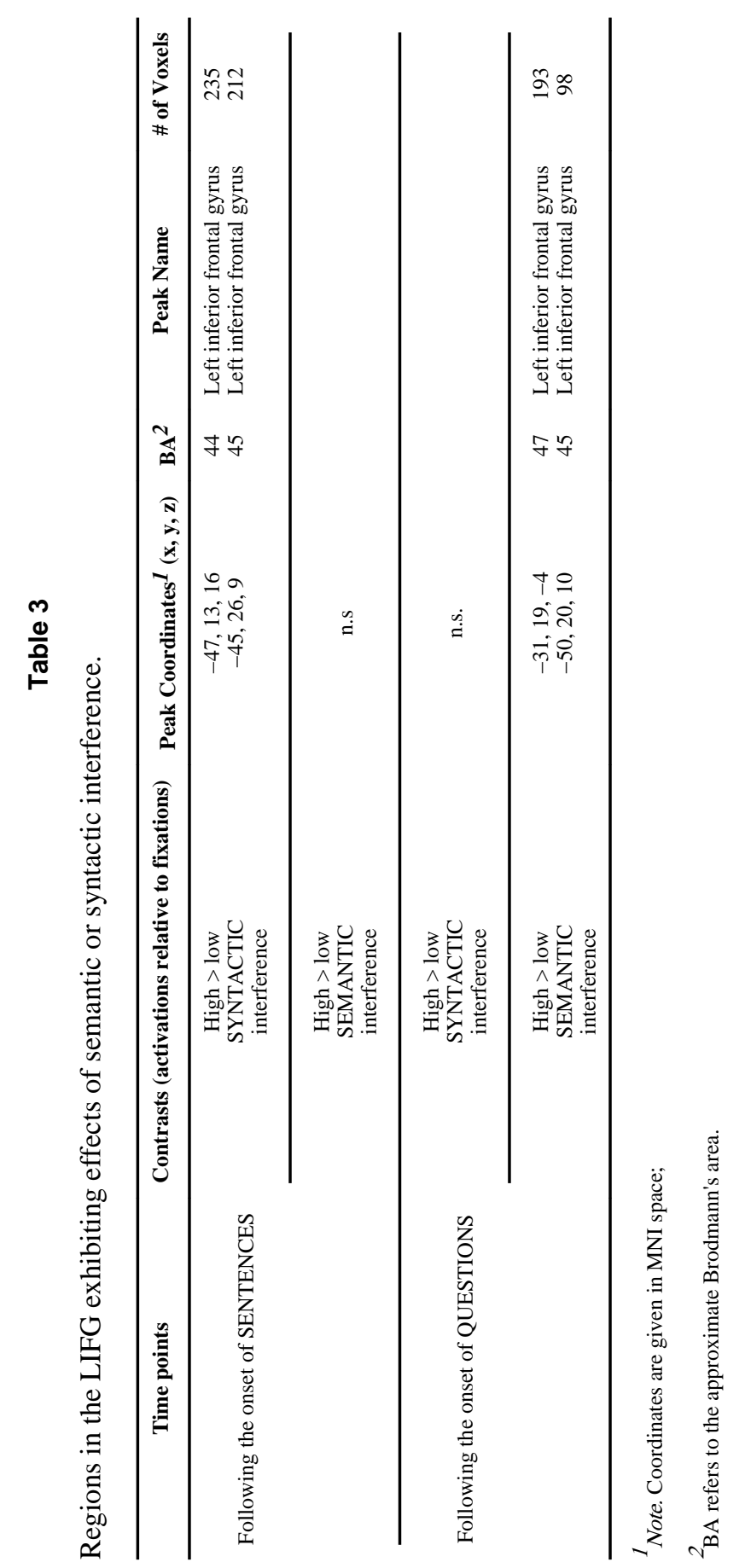

Brain Lang. Author manuscript; available in PMC 2014 September 01. 


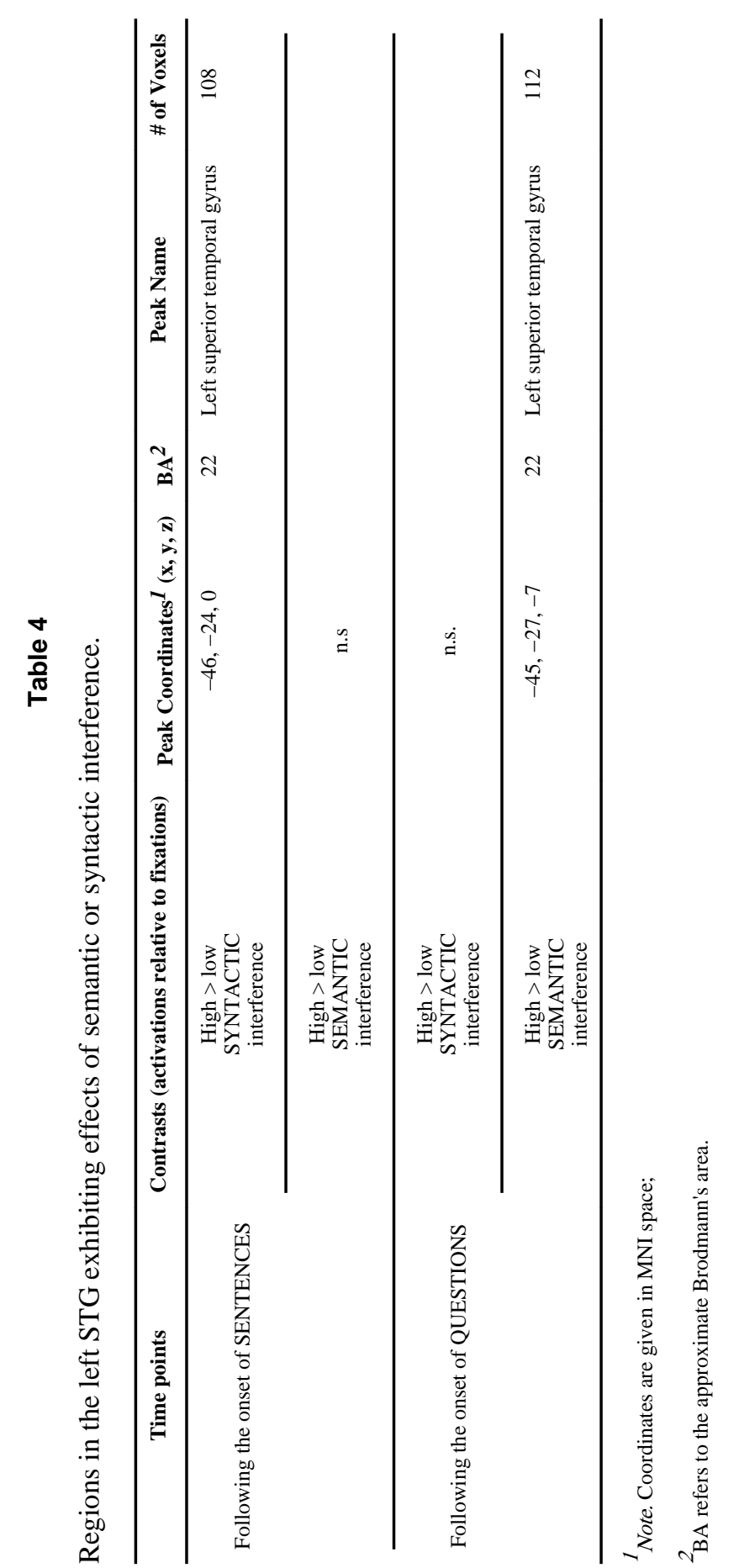

